\title{
DIVERSIDAD DE GÉNERO COMO PARTE DE LA RSC Y SOSTENIBILIDAD EN LAS EMPRESAS. AVANCES PARA LA MEJORA DE LA DIVULGACIÓN DE INFORMACIÓN NO FINANCIERA SOBRE LAS POLÍTICAS DE IGUALDAD Y SUS RESULTADOS
}

Gender Diversity as part of CSR and Sustainability in Companies. Advances for Improving the Disclosure of Non-Financial Information On Equality Policies And Results

\author{
Ainhoa Saitua Iribar \\ Facultad de Relaciones Laborales y Trabajo Social \\ Universidad del País Vasco/Euskal Herrriko Unibertsitatea
}

Orcid: $0000-0002-6937-1753$

Recibido: 30-10-2017

DOI: $10.1387 /$ lan-harremanak. 18897

Aceptado: 11-12-2017

\section{ABSTRACT}

El número cinco de los 17 Objetivos de Desarrollo Sostenible (ODS) de las Naciones Unidas, tal como se aprobaron en 2015, consiste en lograr una igualdad de género efectiva, objetivo que debe promoverse también en el ámbito empresarial. Por otra parte, cada vez es mayor la demanda de transparencia sobre los impactos que genera la actividad de las organizaciones y sobre la propia gobernanza de éstas, como parte de la Responsabilidad Social Corporativa (RSC). Este artículo revisa las principales iniciativas que se están dando para avanzar en la normalización de la divulgación sobre politicas de igualdad en las organizaciones, especialmente, la Comunicación de la Comisión Europea (CE, 2017b), de directrices o guía para ayudar a las empresas que deban aplicar la Directiva 2014/95/EU por primera vez en 
2018. Si bien, en la medida en que la aplicación de la guía será voluntaria, no está garantizada su aplicación por parte de las empresas. También se revisa la guia publicada por la red para liderazgo de la dirección financiera Accounting for Sustainability (A4S, 2017). Como resultado se constata que los ejemplos que ofrecen las empresas que pueden actuar como líderes en la divulgación de politicas de diversidad de género son aún muy escasos. Por lo tanto, creemos que es necesario seguir trabajando en este área que, hasta el presente, no se ha abordado suficientemente, ni en la literatura contable ni en la práctica de las empresas.

Palabras clave: RSC; divulgación de información social; capital humano; género.

Nazio Batuek 2015 an onartu zituzten Garapen Iraunkorrerako 17 helburuetako bostgarrena Genero-berdintasun eraginkorra lortzean datza. Eta helburu hori negozioen alorrean ere sustatu behar da. Bestetik, Gizarte Erantzukizun Korporatiboaren (GEK) eremuan, erakundeen jarduerak sortzen dituzten eraginei buruzko eta beren gobernuari buruzko gardentasun eskaria handituz doa. Artikulu honek enpresa eta bestelako erakundeetako berdintasun politikei buruzko informazioa ematearen normalizazioak aurrera egiteko ematen ari diren ekimen nagusiak berrikusten ditu. Batez ere, $2017 k o$ uztailean Europako Batzordeak onartutako Komunikazioa (CE, 2017b), enpresei laguntzeko, 2018an 2014/95/EB Zuzentaraua lehen aldiz jarraitu beharko dutenean. Edonola ere, gida hori borondatezkoa den neurrian, bere aplikazioa ez dago bermatuta. Bestetik, Accounting for Sustainability finantza zuzendaritzaren lidergorako sareak argitaratutako gida (A4S, 2017) ere berrikusten da. Hemen, genero aniztasun politikei buruzko informazioa emateko lider gisa jardun dezaketen enpresek eskaintzen dituzten adibideak oraindik oso urriak direla ikusi da. Beraz, uste dugu lan gehiago egin behar dela arlo honetan ez baita jorratu nahikoa, ez kontabilitate literaturan ez eta enpresen praktikan ere.

Hitz gakoak: Gizarte Erantzunkizun Korporatiboa; informazio soziala; giza kapitala; generoa.

The number five of the 17 Sustainable Development Goals that the United Nations approuved in 2015 wants to achieve an effective gender equality. It is an objective that should also be promoted in the business world. On the other hand, there is a growing demand for transparency regarding the impacts generated by the activity of organizations and their own governance, as part of Corporate Social Responsibility (CSR). In this context, this article reviews the main initiatives that are being taken to advance the standardization of the disclosure of equality policies in organizations. Especially the Communication of the European Commission (CE, 2017b), to help the companies that must apply Directive 2014/95/EU for the first time in 2018. Although to the extent that the application of the guide will be voluntary, its application by companies is not guaranteed. The guide published by the Accounting for Sustainability CFO leadership network (A4S, 2017) is also re- 
viewed. As a result it is noted that the examples offered by companies that can act as leaders in the disclosure of gender diversity policies are still very scarce. Therefore, we believe that it is necessary to continue working in this area that has not been sufficiently addressed, neither in the accounting literature nor in the practice of the companies.

Keywords: CSR; social information disclosure; human capital; gender. 
DiVERSIDAd DE GÉNERO COMO PARTE DE LA RSC y SOSTENIBILIDAD EN LAS EMPRESAS. AVANCES PARA LA MEJORA

\section{SUMARIO}

1. Introducción. 2. La información no financiera sobre capital humano y social: 2.1. Marco teórico de la información sobre capital humano; 2.2. Regulación en vigor hasta 2017; 2.3. Normativa aplicable al ejercicio 2017 y siguientes. 3. Iniciativas recientes para avanzar en la normalización de la divulgación sobre capital humano y social, incluyendo la diversidad de género. 4. Conclusiones y reflexiones finales. 5. Referencias bibliográficas. 6. Anexo. Listado de marcos internacionales que la Comisión Europea ha tomado como referencia para la elaboración de la guía sobre información no financiera (CE, 2017: 4).

\section{Introducción}

Los últimos años están siendo muy activos en lo que se refiere al desarrollo de iniciativas que promuevan un avance en la igualdad de género efectiva en las empresas, donde aún se constatan brechas en diferentes áreas como, por ejemplo, la remuneración en la alta dirección (Hutchinson et al., 2017). Según el estudio de Grant Thornton, en España ha habido un avance considerable en el número de empresas sin ninguna mujer en la dirección, pasando de $32 \%$ en 2012 a $22 \%$ en 2017. Además, en los últimos diez años, el número de mujeres directivas ha subido del $17 \%$ al $27 \%$, quedando por encima de la media europea. Pero a este ritmo, la paridad efectiva de hombres y mujeres en puestos de responsabilidad se conseguiría en 2037, lo que indica que las políticas de fomento de la igualdad de oportunidades no han sido suficientes (Grant Thornton, 2017). Además, según explica la Red Española para el Desarrollo Sostenible (REDS), aunque en 2017 España mejora cinco puntos en relación con el ránking de 2016 en cumplimiento de los ODS, colocándose en el puesto 25 de entre 157 países, ha perdido el que mejor puntuación tenía, relacionado con el ODS 5 dedicado a la igualdad de género, por lo que aún queda mucho camino por recorrer (Compromiso Empresarial, 2017).

En el Reino Unido, bajo la premisa de que una fuerza de trabajo equilibrada es buena para los negocios (bueno para la clientela, para la rentabilidad y la cultura del lugar de trabajo, y es cada vez más atractivo para las personas y entidades inversoras), el Financial Reporting Council (FRC) ha actualizado la Carta de Mujeres en Finanzas lanzada en 2016, que refleja la aspiración del gobierno de ver el equilibrio de género en todos los niveles en las empresas de servicios financieros, publicando que ya son más de 160 las entidades firmantes, lo que 
abarca a más de 600.000 personas empleadas. Así, la Carta: i) compromete a las empresas para apoyar la progresión de las mujeres en puestos de responsabilidad en el sector de servicios financieros, centrándose en la línea ejecutiva y en el nivel medio; ii) reconoce la diversidad del sector y que las empresas tendrán diferentes puntos de partida (cada firma debería, por lo tanto, establecer sus propios objetivos e implementar la estrategia correcta para su organización); iii) requiere a las empresas a informar públicamente sobre el progreso para cumplir con estos objetivos internos para apoyar la transparencia y la responsabilidad necesarias para impulsar el cambio (HM Treasury, 2017).

En este sentido, en las últimas décadas ha aumentado la importancia de la información no financiera, tanto para ampliar la información financiera tradicional, aportando otros datos complementarios sobre el desarrollo de los negocios, como para informar sobre los impactos que genera la actividad de las distintas entidades para sus distintos grupos de interés (Moser y Martin, 2012). Así, una demanda de información se ha ido conceptualizado inicialmente en términos de la Responsabilidad Social Corporativa de las empresas (RSC) y otras organizaciones (Moneva y Llena, 1996), y más recientemente, en el ámbito de los Objetivos para lograr un Desarrollo Sostenible (ODS), tanto a nivel mundial, como en Europa, en particular (UE, 2013; CE, 2016).

La Unión Europea (UE, 2014), que señala la divulgación de información social y medioambiental como área prioritaria para fortalecer la atención de los negocios hacia la RSC, en 2014 publicó la Directiva 2014/95/UE del Parlamento Europeo y del Consejo, de 22 de octubre de 2014, por la que se modifica la Directiva 2013/34/UE en lo que respecta a la divulgación de información no financiera e información sobre diversidad por parte de determinadas grandes empresas y determinados grupos, para ser traspuesta a la legislación de los Estados Miembros en el plazo de 2 ańos y aplicada para los ejercicios iniciados a partir del 1 de enero de 2017. Así, las empresas que se consideren de interés público de más de 500 personas empleadas y que cumplan otros criterios para considerase grandes, deberán incluir en el Informe de Gestión, un Estado No Financiero que contenga información sobre las políticas, los resultados de dichas políticas y los riesgos asumidos en cuestiones relativas al medioambiente, los derechos humanos, la lucha contra la corrupción y el soborno, así como al personal, ámbito en el que se informará también sobre las políticas de diversidad, incluidas las relacionadas con el género.

En lo que respecta al objetivo de diversidad en los órganos de dirección de las empresas, el considerando número 18 de la Directiva 2014/95/UE argumenta que "la diversidad de competencias y puntos de vista de los miembros de los órganos de administración, dirección y supervisión de las empresas facilita una buena comprensión de la organización empresarial y de los negocios de la empresa de que se trate. Esa diversidad permite a los miembros de esos órganos ejercer una critica constructiva de las decisiones de la dirección y ser más receptivos a las ideas in- 
novadoras, combatiendo asi el fenómeno del "pensamiento de grupo", caracterizado por la semejanza de los puntos de vista de los miembros. La diversidad contribuye así a una supervisión eficaz de la dirección y a una gobernanza satisfactoria de la empresa. Es importante, pues, mejorar la transparencia con respecto a la politica de diversidad aplicada. Con ello se informaría al mercado de las prácticas de gobierno corporativo y se presionaría asi indirectamente a las empresas para que aumenten la diversidad de sus consejos de administración».

Con estos antecedentes, el objetivo de este artículo consiste en realizar una revisión de las últimas normas y recomendaciones que promueven el avance en la comunicación sobre las políticas para fomentar la diversidad de género real en las empresas y sobre los resultados obtenidos por las mismas. Se analiza, especialmente, la Comunicación de Directrices publicada por la Comisión Europea (CE, 2017b) para ayudar a las empresas a aplicar la Directiva 2014/95/EU por primera vez en 2018. Tras esta introducción, el apartado 2 realiza una breve revisión de la literatura contable sobre divulgación de información relativa al personal, el apartado 3 recoge las iniciativas relevantes más recientes para divulgar información no financiera sobre aspectos sociales y del personal, pero centrando el foco en las propuestas que se hayan realizado para mejorar la información sobre las políticas de diversidad de género y sus resultados, incluyendo algunos ejemplos seleccionados por las propias empresas que pueden estar actuando como líderes en este ámbito, y el último se dedica a las conclusiones y reflexiones finales.

\section{La información no financiera sobre capital humano y social}

\subsection{Marco teórico de la información sobre capital humano}

El capital humano es único, en el sentido de que las personas empleadas por una organización comportan la doble naturaleza de grupo interesado en la información sobre la evolución de la empresa, a la vez que el propio capital humano constituye un recurso o factor interno. Así, en el marco de la Teoría de Recursos y Capacidades, la gestión eficaz de los recursos humanos contribuye a reforzar la competitividad y calidad de la organización (Sainz, 1990), aun más cuando en la actualidad se considera que el capital humano es clave para el éxito del negocio (Gamerschlag y Moeller, 2011). Pero, la contabilidad tradicional no considera al capital humano como un activo en el Balance contable (Dobija, 1998; Sierra y Moreno, 2000) de forma que, en este ámbito, las explicaciones narrativas resultan críticas (Knauer, 2010). En todo caso, en ausencia de normativa contable que las obligue, las empresas han complementado la información sobre los recursos humanos mediante revelación voluntaria de información (GarcíaMeca, 2008; Wyatt y Frick, 2010). Y diversos estudios han obtenido evidencias de que la divulgación sobre capital intelectual y recursos humanos presenta una relación positiva con el valor de la empresa (Ghasempour y Yusof, 2014), si bien sugieren que los beneficios que se producen por las actividades de RSC y su di- 
vulgación no benefician únicamente a los inversores, sino a un grupo más amplio de grupos de interés (Moser y Martin, 2012).

La literatura contable ha propuesto distintas formas para superar la limitación de la contabilidad de la inclusión del capital humano como activo en los balances, hasta llegar a un sistema de indicadores en diferentes tipos de memorias narrativas (Sierra y Moreno, 2000; Martínez y Burgos, 2010). Así, a nivel internacional, la divulgación narrativa sobre los recursos humanos puede incluirse dentro del informe anual, tanto en la Memoria que forma parte de las cuentas anuales y en el Informe de Gestión que las acompańa, como en los informes de RSC que publican algunas entidades de forma voluntaria, o en otros soportes como notas de prensa, conferencias a analistas, etc. Sin embargo, el área de la información narrativa sobre las personas empleadas por la empresa es la que está menos desarrollada dentro de la divulgación corporativa (Mäkelä, 2010) y en todas las corrientes teóricas Contabilidad de los Recursos Humanos (CRH) (Marqués, 1974; Martínez Churiaque 1978, 1980; Villacorta, 2006); Capital Intelectual (CI) (Méritum 1998, 2002; AECA, 2012), o Contabilidad Social (Pons, 1996), aparece como problema la falta de regulación de contenidos y formatos que, a la postre, reduce la relevancia (Saitua et al., 2014a) y comparabilidad (Saitua et al., 2014b) y, por tanto, la utilidad de la información divulgada (Carmona y Carrasco, 1988; Moneva y Llena, 1996; Archel, 2003; Villacorta, 2006; Aranguren y Ochoa, 2008).

\subsection{Regulación del Informe de Gestión en vigor hasta 2017}

En España, a partir de la incorporación de las antiguas IV y VII directivas contables europeas ${ }^{1}$ mediante la Ley 19/1989, de 25 de julio, de reforma parcial y adaptación de la legislación mercantil a las Directivas de la comunidad Económica Europea (CEE) en materia de Sociedades, la legislación mercantil regula el Informe de Gestión como un documento que acompaña a las Cuentas Anuales, y que informa sobre la evolución de la situación económica, financiera y de los resultados de una empresa. Según se desprendía del artículo 202 del Texto Refundido de la Ley de Sociedades Anónimas (en la actualidad en el artículo 262 de la Ley de Sociedades de Capital por RDL 1/2010), en su texto vigente hasta 2004, el Informe de Gestión debía contener: a) una exposición fiel sobre la evolución de los negocios y situación de la sociedad; b) información sobre los acontecimientos importantes ocurridos después del cierre del ejercicio; c) información sobre la evolución previsible de la sociedad; d) las actividades realizadas en materia de inves-

${ }^{1}$ Cuarta Directiva del Consejo de 25 de julio de 1978 basada en la letra g) del apartado 3 del artículo 54 del Tratado y relativa a las cuentas anuales de determinadas formas de sociedad (78/660/ CEE, DO L 222 de 14.8.1978, p. 11) y Séptima Directiva del Consejo de 13 de junio de 1983 basada en la letra g) del apartado 3 del artículo 54 del Tratado, relativa a las cuentas consolidadas (83/349/CEE), derogadas por la Directiva 2013/34/UE, que actualizó su contenido. 
tigación y desarrollo; e) las adquisiciones de acciones propias. De esta manera, el contenido del informe no estaba suficientemente regulado, pues no se establecían los objetivos que se perseguían con la elaboración del mismo, ni se daban ejemplos del nivel de detalle que debería ofrecerse para cada ítem, dando a las empresas la opción de cumplir un mínimo formal y restando utilidad al documento, de modo que requería un mayor desarrollo normativo (ICAC 2002: 151). Posteriormente, en transposición de la Directiva 2003/51/CE del Parlamento Europeo y del Consejo, de 18 de junio de 2003, sobre las cuentas anuales y consolidadas de determinadas formas de sociedades, bancos y otras entidades financieras y empresas de seguros (Directiva de Modernización). el contenido del Informe de Gestión fue ampliado por la Ley 62/2003, de 30 de diciembre, de medidas fiscales, administrativas y del orden social, que incluyó, como contenidos a divulgar a partir del ejercicio 2005, la identificación de los principales riesgos e incertidumbres a los que se enfrenta la sociedad, así como la utilización de indicadores financieros y no financieros cuando sean necesarios para la comprensión de la evolución, los resultados o la evolución de la sociedad. Además, en la medida en que sean relevantes, se incluyó la obligación de informar sobre cuestiones relativas al medio ambiente y al personal, que algunas empresas ya venían revelando en este documento o en otros, como los informes de Responsabilidad Social, de forma voluntaria.

\section{Cuadro 1}

Propuesta para la recogida de información

sobre los tipos de contenido divulgado en relación con el personal y la diversidad, en base a las recomendaciones de la guía CNMV (2013)

\begin{tabular}{|c|c|c|}
\hline $\begin{array}{l}\text { Estructura } \\
\text { del informe } \\
\text { de gestión }\end{array}$ & $\begin{array}{l}\mathrm{N}^{\circ} \\
\text { recom. }\end{array}$ & Indicadores sobre contenido \\
\hline $\begin{array}{l}2.1 \text { Indicadores } \\
\text { fundamentales } \\
\text { de carácter } \\
\text { financiero y no } \\
\text { financiero }\end{array}$ & 6 & $\begin{array}{l}\text { Para cada indicador: } \\
\text { 1. ¿Informa sobre su definición? } \\
\text { 2. ¿Informa sobre el propósito perseguido a través de su uso? } \\
\text { 3. ¿Informa sobre el método de cálculo y valor, con la información } \\
\text { comparativa correspondiente? } \\
\text { 4. ¿Informa sobre la relación existente entre el indicador y el propósito } \\
\text { para el cual se utiliza? } \\
\text { 5. ¿Revela los cambios relevantes en la construcción de los indicadores? } \\
\text { 6. ¿Explica los cambios relevantes en la construcción de los indicadores? } \\
\text { 7. Cuando se produzcan estos cambios, es recomendable que, si fuera } \\
\text { posible y al objeto de proveer una comparación entre el ejercicio so- } \\
\text { bre el que se reporta y el anterior, se ofrezca un indicador proforma } \\
\text { que sustituya al presentado en el informe de gestión anterior, intro- } \\
\text { duciendo la modificación que se haya producido en este ejercicio. } \\
\text { :Se informa sobre ese indicador proforma? }\end{array}$ \\
\hline
\end{tabular}




\begin{tabular}{|c|c|c|}
\hline $\begin{array}{l}\text { Estructura } \\
\text { del informe } \\
\text { de gestión }\end{array}$ & $\begin{array}{l}\mathrm{N}^{\circ} \\
\text { recom. }\end{array}$ & Indicadores sobre contenido \\
\hline $\begin{array}{l}\text { 2.2. Cuestiones } \\
\text { relativas al } \\
\text { medioambiente } \\
\text { y al personal } \\
\text { 2.2.2.Personal }\end{array}$ & 10 & 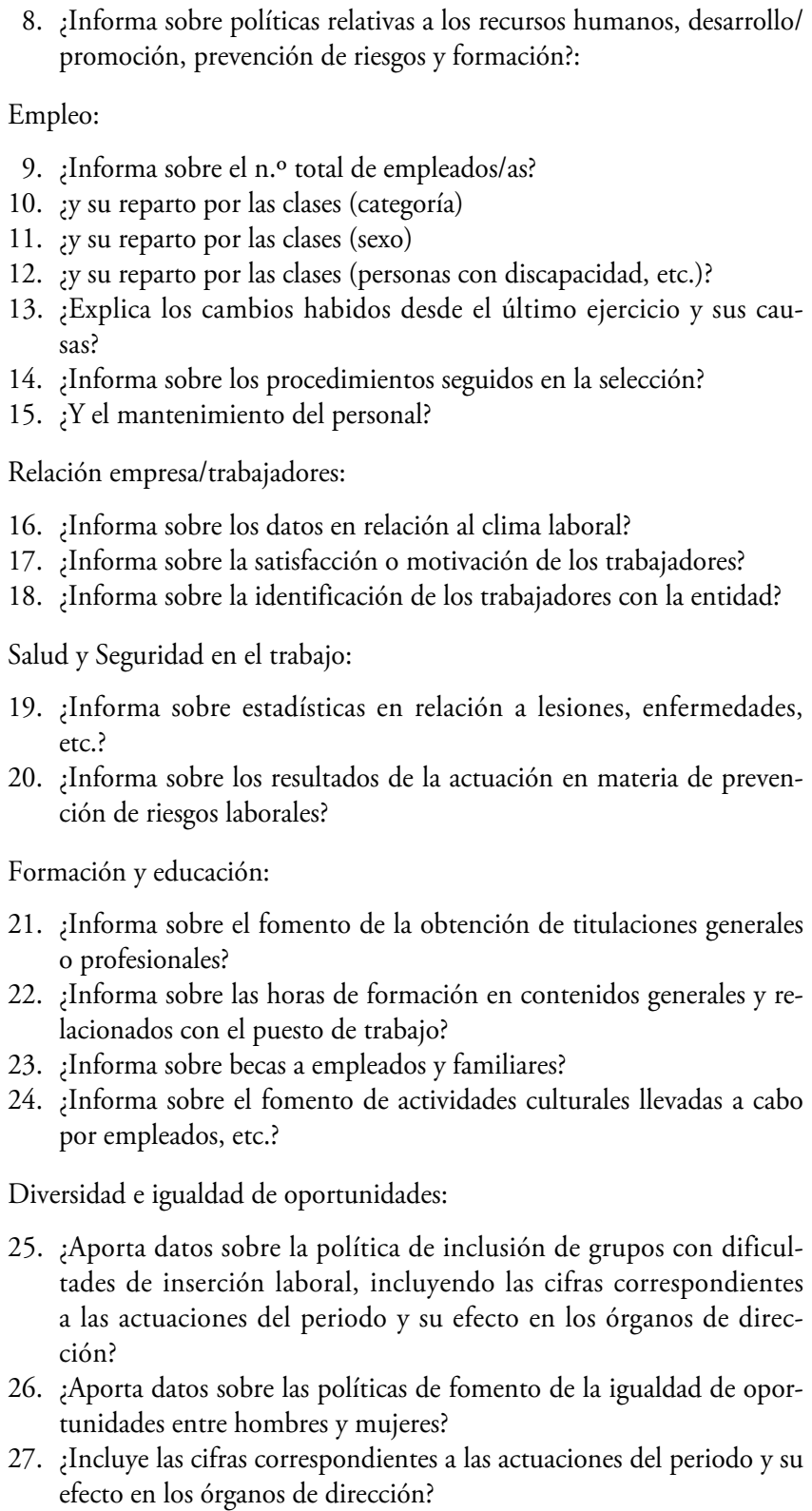 \\
\hline
\end{tabular}

Fuente: elaboración propia a partir de Saitua et al. (2015). 
Por otra parte, en 2013, la Comisión Nacional del Mercado de Valores publicó una Guía para el Informe de Gestión de las empresas cotizadas españolas con el ánimo de mejorar la calidad de la información que presentan estas empresas. A continuación mostramos un listado de indicadores que pueden ser de utilidad, tanto con fines de investigación para analizar el nivel de información divulgado, como para las propias entidades, cotizadas y no cotizadas, que quieran cumplir con los requisitos de calidad recomendados a la hora de preparar sus Informes de Gestión basándose en dicha guía (CNMV, 2013), en relación con la información sobre el personal y sobre la diversidad.

$\mathrm{Al}$ no ser de aplicación obligatoria, la implementación de esta guía (CNMV, 2013) no está garantizada por parte de las empresas, pero en cualquier caso, supone un paso adelante en la normalización de las prácticas de divulgación para todas aquellas empresas cotizadas o no cotizadas que quieran utilizarla como orientación en elaboración de un Informe de Gestión de calidad.

\subsection{Normativa aplicable al ejercicio 2017 y siguientes}

A partir del desarrollo de diferentes comunicaciones sobre transparencia y RSC emitidas por la Unión Europea desde 2011 en adelante, el 6 de diciembre de 2014 entró en vigor la Directiva 2014/95/EU sobre divulgación de información no financiera e información sobre diversidad para ciertas grandes entidades y grupos de más de 500 personas empleadas, que la aplicarán en 2018 para informar sobre el ejercicio 2017. Se espera que una mayor transparencia logre que las empresas rindan mejor y sean más resilientes, tanto en términos financieros como no financieros, pero los requisitos no se aplican a empresas de menor tamaño, porque se entiende que sus costes podrían superar los beneficios de una mayor información.

Así, la situación respecto de la información sobre el personal y diversidad en el Informe de Gestión en el ámbito de la Unión Europea sería el siguiente, dependiendo del tipo de empresa.

Toda esta regulación de la información exigida es equivalente para los grupos de sociedades, con sus disposiciones correspondientes para los Informes de Gestión consolidados.

En España, aunque con retraso, ya se ha transpuesto el contenido de la Directiva 2014/95/UE, modificando el artículo 49 del Código de Comercio, así como los artículos 262 y 540 del Texto Refundido de la Ley de Sociedades de Capital, y la Ley de Auditoría de Cuentas. En enero de 2017 el ICAC (Instituto de Contabilidad y Auditoría de Cuentas) inició un período de información pública sobre el Anteproyecto de Ley, que finalmente en septiembre fue aprobado por el Consejo de Ministros para ser remitido al Consejo de Estado y recibir dictamen y posterior tramitación parlamentaria, si bien debido a la amenaza de 


\section{Cuadro 2}

Regulación de la información sobre personal y diversidad en el Informe de Gestión en la Unión Europea

\begin{tabular}{|c|c|c|c|}
\hline $\begin{array}{c}\text { Directiva } \\
\text { 2013/34/UE } \\
\text { (que deroga } \\
\text { las Directivas } \\
\text { 78/660/CEE } \\
\text { y 83/349/CEE) }\end{array}$ & $\begin{array}{l}\text { Empresas } \\
\text { que no } \\
\text { pueden } \\
\text { presentar } \\
\text { Balance } \\
\text { Abreviado }\end{array}$ & $\begin{array}{l}\text { Entidades de } \\
\text { interés público con } \\
\text { número medio de } \\
\text { personas empleadas } \\
>500 \text { y cumplan } \\
\text { otros criterios para } \\
\text { considerarse grande }\end{array}$ & $\begin{array}{l}\text { Entidades de interés público con valores admitidos a } \\
\text { cotización (cotizadas) }\end{array}$ \\
\hline Art. 19 & \multicolumn{3}{|c|}{$\begin{array}{l}\text { INFORME DE GESTIÓN: En la medida necesaria para la compresión de la } \\
\text { situación de la empresa..., incluirá indicadores financieros y no financieros... } \\
\text { incluida información relativa al medioambiente y al personal. }\end{array}$} \\
\hline Art. 19 bis* & - & \multicolumn{2}{|c|}{$\begin{array}{l}\text { ESTADO NO FINANCIERO* en el Informe de Gestión (o en } \\
\text { un informe separado): En la medida necesaria para la compresión } \\
\text { de la situación de la empresa... y el impacto de su actividad rela- } \\
\text { tiva, como mínimo, a cuestiones medioambientales y sociales, así } \\
\text { como relativas al personal, al respeto de los derechos humanos y a } \\
\text { la lucha contra la corrupción y el soborno, y que incluya: ... des- } \\
\text { cripción de las políticas que aplica en relación con dichas cuestio- } \\
\text { nes, que incluya los procedimiento de diligencia debida aplicados; } \\
\text { los resultados de esas políticas; los principales riesgos.. y cómo los } \\
\text { gestiona; indicadores clave de resultados no financieros... y si no } \\
\text { se aplica ninguna política sobre una o varias de esas cuestiones, } \\
\text { una explicación clara y motivada al respecto. }\end{array}$} \\
\hline Art. 20 & - & - & $\begin{array}{l}\text { DECLARACIÓN DE GOBERNANZA EM- } \\
\text { PRESARIAL en el Informe de Gestión: Có- } \\
\text { digo de gobernanza al que esté sujeta, código } \\
\text { que haya decidido aplicar, prácticas de gober- } \\
\text { nanza, características de los sistemas de con- } \\
\text { trol interno y gestión de riesgos en relación } \\
\text { con el proceso de emisión de información fi- } \\
\text { nanciera, etc. }\end{array}$ \\
\hline $\begin{array}{l}\text { Art. } 20 \\
\text { aptdo. } 1 \\
\text { letra g)* }\end{array}$ & - & - & $\begin{array}{l}\text { DESCRIPCIÓN DE LA POLÍTICA DE } \\
\text { DIVERSIDAD* (por ejemplo, sobre edad, } \\
\text { formación y experiencia profesional o género) } \\
\text { para los órganos de administración, dirección } \\
\text { y supervisión: objetivos de la política; cómo se } \\
\text { ha aplicado; resultados; y si no se aplica nin- } \\
\text { guna política, explicación al respecto. }\end{array}$ \\
\hline
\end{tabular}

* Añadido por art. 1 de la Directiva 2014/95, para aplicar en 2018 a la información sobre el ejercicio 2017.

Fuente: elaboración propia. 
recibir una sanción por parte del Tribunal de Justicia de la Unión Europea, ha sido aprobado de forma urgente mediante el Real Decreto-ley 18/2017, de 24 de noviembre por el que se modifican el Código de Comercio, el texto refundido de la Ley de Sociedades de Capital aprobado por el Real Decreto Legislativo 1/2010, de 2 de julio, y la Ley 22/2015, de 20 de julio, de Auditoría de Cuentas, en materia de información no financiera y diversidad.

Este RDL 18/2017 establece que las Entidades de Interés Público en España presentarán un Estado No Financiero (individual o consolidado) si el número medio de personas empleadas (o del grupo) es superior a 500, y si durante dos ejercicios consecutivos se cumplen dos de estas tres circunstancias: a) Activo total (o consolidado) superior a 20.000.000 euros; b) Importe Neto de la cifra de Negocios (o consolidada) supere los 40.000 .000 euros; c) Número medio de personas empleadas de la entidad (o de la dominante) sea superior a doscientos cincuenta.

De modo que las empresas que cumplan dichos criterios de tamaño se enfrentan a un reto mayor de preparación de información sobre las políticas y los resultados relacionados con diversas cuestiones entre las que se encuentran el personal, los derechos humanos y la lucha contra la corrupción y el soborno.

\section{Iniciativas recientes para avanzar en la normalización de la divulgación sobre capital humano y social, incluyendo la diversidad de género}

En los últimos años están aumentando las iniciativas públicas y privadas que incluyen como uno de sus aspectos a tratar, tanto la diversidad de género, como su divulgación. Por ejemplo, la Carta de Mujeres en Finanzas citada anteriormente, recoge que cada organización se compromete concretamente a (HM Treasury, 2017):

— tener un miembro del equipo ejecutivo senior responsable de la diversidad de género y la inclusión;

- establecer objetivos internos para la diversidad de género en la alta gerencia;

- tener la intención de asegurar que la remuneración del equipo ejecutivo sénior esté vinculado al logro de estos objetivos internos sobre la diversidad de género;

- publicar anualmente el progreso de estos objetivos en los informes en el sitio web de la empresa.

Por su parte, la Guia Esencial para la Contabilidad Social y del Capital Humano que se reconoce como elaborada por equipos financieros (entre otras, han participado personas directivas de Siemens, Burberry o Sainsbury's) para ayudar a equipos financieros, mediante ejemplos y otra serie de herramientas, presenta este listado de temas que si bien no es exhaustivo, muestra las áreas clave que, en 
su experiencia, se encuentran en el ámbito de las grandes definiciones sobre capital humano y social (A4S, 2017: 6).

Licencia para operar:

- Confianza y reputación.

- Respuesta a las expectativas sociales.

- Política fiscal responsable.

— Relaciones con la comunidad.

Justicia económica:

- Acceso a los productos y servicios básicos como el combustible, agua, sanidad, medicinas, servicios financieros, comunicaciones, etc.

-Asequibilidad.

Tendencias sociales:

— Envejecimiento de la población.

— Obesidad y nutrición.

- Urbanización.

- Ciudadanía.

— Inclusión: género, raza, religión, orientación sexual, personas económicamente desfavorecidas.

Fuerza y resiliencia de la fuerza de trabajo:

- Atracción, selección y retención.

- Creación de empleo.

- Capacidades.

- Formación y educación.

- Personal en formación.

Condiciones de trabajo:

- Reconocimiento y remuneración justa.

- Comercio justo.

- Trabajo forzado.

- Trabajo infantil.

- Derechos laborales.

— Libertad de asociación y derecho a la negociación colectiva.

— Salud y seguridad.

- Diversidad.

- Horario laboral.

- Bienestar.

Derechos Humanos:

- Privacidad.

— Seguridad, incluida la seguridad cibernética.

— Libertad de expresión. 
Además, la guía explica que las organizaciones pueden identificar tanto impactos como dependencias entre el capital humano y social. Por ejemplo, una empresa puede impactar en la empleabilidad de su personal a través de su enfoque de formación y puede ser dependiente de una adecuada provisión de reclutamientos adecuadamente educados y formados (AS4, 2017). En este sentido, observamos que en lo que respecta a la variable género (la incorporación de las mujeres), la empresa produce impactos, por una parte, en la «fuerza y resiliencia de la fuerza de trabajo», en la medida que algunas de las capacidades de que dispongan las mujeres sean diferentes de las de los hombres, y especialmente en el área "condiciones de trabajo» de las entidades, en el sentido de que aumenta su «diversidad». Pero entendemos que las empresas deben cuidar también las áreas de "Reconocimiento y remuneración justa», «Derechos laborales», «Seguridad y salud», y en definitiva, "Bienestar» de todas las personas, para que no se produzcan discriminaciones o brechas entre mujeres y hombres.

En todo caso, la guía recuerda que el rol del equipo financiero es clave para asegurar que el modelo financiero puede ser validado, y soportado en evidencias, incluso asegurado externamente (A4S 2017: 57). Entre las empresas que han participado, el ejemplo de SSE (Scotish and Southern Energy) destaca por el desarrollo del modelo que les ha permitido aumentar la comprensión del valor de su capital humano y del impacto que tiene en su rendimiento empresarial. Entre otros temas, esta empresa señala como uno de los retos del sector la falta de diversidad, en la medida en que solo el 15\% de los empleos corresponde a mujeres (A4S, 2017: 90).

Entre otros ejemplos de las empresas participantes, Unilever (A4S, 2017: 12) señala el rol de su compromiso social en el desarrollo de nuevos mercados. Una vez que identifican a las mujeres como el $70 \%$ de las personas consumidoras, y también como participantes activas en la cadena de valor (granjeras, empleadas, distribuidoras), entienden que empoderar económicamente a las mujeres crea un efecto en las familias, las comunidades y las economías. Afirman saber intuitivamente el valor de empoderar a la mujer, pero advierten de que sin una métrica sólida para analizar la inversión, ésta podría no ser tan efectiva como debería. En concreto, han definido un compromiso para empoderar 5 millones de mujeres para 2020, especialmente en áreas donde quieren crecer, como África. Así, midiendo el compromiso de empoderar a las mujeres, evidencian tanto el crecimiento de oportunidades de sus marcas como la contribución para generar mayores oportunidades a las mujeres. A modo de ejemplo, citan un proyecto de aprendizaje online de mujeres en la India (A4S, 2017: 55)

Pero en la guía (A4S, 2017) no encontramos ningún ejemplo de información sobre políticas de capital humano relativos a la diversidad de género, esto es, de objetivos que se hayan planteado (con sus correspondientes criterios a aplicar, procedimientos de verificación, etc.) para aumentar la diversidad y sus relaciones con los procesos de selección, formación, remuneración, etc. de las mujeres empleadas. 
Cuadro 4

Ejemplo práctico de Unilever

\begin{tabular}{|c|c|c|c|}
\hline Beneficio para el negocio & $\begin{array}{l}\text { Métrica y fuente } \\
\text { de información }\end{array}$ & Impacto. Dependencia & $\begin{array}{l}\text { Relación con otros } \\
\text { capitales }\end{array}$ \\
\hline $\begin{array}{l}\text { - Demostrar progreso } \\
\text { en el compromiso. } \\
\text { — Lograr visión de } \\
\text { crecer el negocio de } \\
\text { forma sostenible. } \\
\text { - Mejorar reputación } \\
\text { como líder de sos- } \\
\text { tenibilidad. }\end{array}$ & $\begin{array}{l}\text { - Número de muje- } \\
\text { res que participan } \\
\text { en iniciativa de me- } \\
\text { jora de capacidades } \\
\text { (n. de asistencias). } \\
\text { - Número de muje- } \\
\text { res empoderadas } \\
\text { (encuesta de satis- } \\
\text { facción). }\end{array}$ & $\begin{array}{l}\text { Impacto: } \\
\text { — Nuevos mercados. } \\
\text { — Mejora de capaci- } \\
\text { dades. } \\
\text { - Disminución de in- } \\
\text { cidentes de salud. } \\
\text { Dependencia: } \\
\text { - Reputación y con- } \\
\text { fianza. } \\
\text { - Nivel de capacida- } \\
\text { des locales. }\end{array}$ & $\begin{array}{l}\text { Capital Financiero: } \\
\text { — Aumento de ingre- } \\
\text { sos. } \\
\text { - Nuevos mercados. } \\
\text { — Valor de marca. } \\
\text { Capital Humano: } \\
\text { - Aumento de dere- } \\
\text { chos. } \\
\text { - Mejora de capaci- } \\
\text { dades. } \\
\text { - Mejora de oportu- } \\
\text { nidades. }\end{array}$ \\
\hline
\end{tabular}

Fuente: tomado de A4S (2017: 56).

Finalmente, la Directiva 2014/95/UE en su artículo 2 se comprometió a que antes de diciembre de 2016 la Comisión preparara unas directrices no vinculantes sobre la metodología aplicable a la presentación de información no financiera, que incluyeran indicadores clave de resultados no financieros, de carácter general y sectorial, con el fin de facilitar la divulgación pertinente, útil y comparable de información no financiera por parte de las empresas. Así, en desarrollo del artículo 2, y tras un proceso de consulta pública ${ }^{2}$, también con retraso sobre los plazos inicialmente previstos, la Comisión Europea publicó un borrador titulado Guidelines on non-financial reporting (methodology for reporting non-financial information) (CE 2017a), que finalmente se aprobó como Comunicación en julio de 2017 (CE, 2017b).

Si bien la presentación de un Estado No Financiero puede realizarse de forma alternativa (en aplicación del artículo 1 de la Directiva 2014/95/UE), esta guía lo sitúa en el contexto de un Informe de Gestión (Management Report). Con una metodología basada en principios que sirva para empresas de todos los sectores de actividad, la guía se ha basado en las mejores prácticas y desarrollos relevantes que se están produciendo tanto a nivel de la UE como a nivel internacional (ver Anexo). Entre ellos destacamos la Global Reporting Initiative

\footnotetext{
${ }^{2}$ La propia guía incluye una nota al pie para remitir a más información sobre el proceso de consulta pública a través del siguiente enlace: http://ec.europa.eu/finance/company-reporting/nonfinancial_reporting/index_en.htm\#related-documents
} 
(GRI) cuya última versión fue analizada por Moneva et al. (2014), o el International Integrated Reporting Framework <IR>, tal como se propone en Saitua et al. (2014c). Pero echamos en falta que no hayan citado el proyecto del Management Comentary del IASB (International Accounting Standards Board), utilizado como referencia para el estudio de Saitua et al. (2014a).

Esta guía (CE, 2017b) contiene seis apartados, de los que comentamos los que nos resultan de mayor interés en relación con la divulgación sobre diversidad:

1. Introducción.

2. Objetivo

3. Principios fundamentales:

3.1. Divulgación de información significativa.

3.2. Imagen fiel, equilibrada y comprensible.

3.3. Información completa pero concisa.

3.4. Información estratégica y prospectiva.

3.5. Información orientada a las partes interesadas.

3.6. Información coherente y sistemática.

4. Contenido:

4.1. Modelo de negocio.

4.2. Políticas y debida diligencia.

4.3. Resultados.

4.4. Principales riesgos y su gestión.

4.5. Principales indicadores de resultados.

4.6. Cuestiones específicas.

5. Marcos de presentación de informes.

6. Información sobre la diversidad del Consejo de Administración.

La introducción cita tanto el objetivo general de Estabilidad Financiera en el marco de los acuerdos del G20 y los bancos centrales, como el acuerdo de la Comisión Europea (CE, 2016) para responder a la Agenda 2030 adoptada por las Naciones Unidas en septiembre de 2015, de la que se cita expresamente el objetivo 5.5: "Velar por la participación plena y efectiva de las mujeres y la igualdad de oportunidades de liderazgo a todos los niveles de la adopción de decisiones en la vida politica, económica y pública».

En lo que se refiere al principio fundamental de divulgación de información significativa (subapartado 3.1 de la guía), explica que los impactos de la actividad de una empresa pueden ser positivos o adversos, y que se espera que la información no financiera ofrezca una imagen fiel de la información que necesitan 
las personas y entidades consideradas como stakeholders de dicha entidad. Por otra parte, dentro de un mismo sector de actividad es posible que se enfrenten a retos similares y, por lo tanto, puede ser apropiado que se comparen las divulgaciones entre empresas del mismo sector.

El subapartado 4.6, titulado "Cuestiones específicas», contiene un epígrafe b) Cuestiones Sociales y relativas al Personal», que incluye información sobre gestión del capital humano, relaciones sindicales, etc., así como sobre diversidad (edad, étnica, etc. incluyendo la de género) e igualdad de trato en el empleo y la ocupación. En este ámbito, como ejemplos de Indicadores Clave de Resultados que una entidad podría considerar divulgar, figuran (CE 2017b: 16):

i) Diversidad de género.

ii) Derecho al permiso parental, por sexo.

iii) Temporalidad laboral, por sexo.

iv) Promedio de horas de formación por persona empleada, por sexo.

En cuanto a la divulgación sobre la diversidad del Consejo de Administración, el apartado 6 de esta guía ofrece una orientación específica para ayudar a las grandes compañías cotizadas para que preparen una descripción de su política de diversidad del Consejo, que deberá ser incluIda (recordando que esta no forma parte del Estado No Financiero), en su Informe de Gobierno Corporativo a partir de 2018, lo que no obsta para que la empresa pueda incluir información relevante sobre diversidad como parte del Estado No Financiero. En primer lugar, la guía cita el contenido normativo de la nueva Directiva 2014/95/UE, de forma que las entidades cotizadas deben incluir en su Informe de Gobierno Corporativo:

— una descripción de la política de diversidad en relación con los órganos de administración, gestión y supervisión relativa a aspectos como por ejemplo, la edad, la formación académica, experiencia profesional, o el género;

— los objetivos de dicha política;

— cómo ha sido implementada,

— los resultados en el periodo del que se informa.

Y si no se aplicara, el Informe incluirá una explicación de por qué es el caso.

En lo que se refiere a los aspectos de diversidad (que en principio cubren la edad, género, y experiencia educacional y profesional, pero pueden incluir origen geográfico, experiencia internacional, capacidades en aspectos de sostenibilidad, representación de personas empleadas, u otros), la guía propone que la política debería informar sobre los siguientes aspectos:

- Criterios. Cuáles se aplican (para garantizar que el Consejo tiene una diversidad suficiente de puntos de vista y experiencia necesaria para una 
buena comprensión de los asuntos actuales, así como los riesgos y oportunidades a lo largo plazo relacionados con el negocio), y

- Razón para elegirlos. Así, en la selección de una persona candidata en base a los criterios de diversidad que se hayan definido, se deberían tener en cuenta las normas y principios generalmente aceptados de no discriminación.

- Objetivos. Las empresas deberían divulgar objetivos específicos medibles para los aspectos relevantes de la diversidad. La guía advierte que es particularmente útil establecer objetivos cuantitativos y marcos temporales, en particular, respecto del equilibrio de género.

- Implantación de políticas de diversidad. La guía sugiere que las empresas deberían indicar cómo se tienen en consideración los objetivos de la política de diversidad en el plan de sucesión, la selección, la nominación y la evaluación.

— Rol de los comités competentes en estos procesos.

- Información sobre criterios de diversidad y sus objetivos. Las empresas deberían divulgar si se ha proporcionado a las personas y entidades accionistas en el momento de elegir o renovar las personas miembros del Consejo, cuando sea relevante.

— Estado de la implementación y resultados desde el último informe para todos los aspectos de diversidad. Si los objetivos de diversidad no se han cumplido, la empresa debería divulgar cómo pretende cumplir los objetivos, incluyendo el marco de tiempo esperado en el que dichos objetivos deben cumplirse.

En todo caso, el objetivo de la guía es ayudar a las entidades afectadas a divulgar información no financiera de una forma relevante, útil, consistente y más comparable, pero las entidades que utilicen esta guía también pueden basarse en otros marcos nacionales (como el de la CNMV, 2013), europeos o internacionales. Se trata de una guía no vinculante, de forma que no crea nuevas obligaciones legales. De este modo, el documento no constituye una norma técnica. Por lo tanto, ni las entidades preparadoras ni otras partes interesadas deberían reivindicar que el Estado No Financiero ha sido preparado de conformidad con dicho documento. Por último, si bien el documento se dirige a las entidades que deben aplicar la Directiva 2014/95/EU, la guía representa un modelo de buenas prácticas para todas las empresas o entidades que divulgan información no financiera, incluidas las que no se encuentran en el ámbito de aplicación de la Directiva (CE 2017b: 4). 


\section{Conclusiones y reflexiones finales}

Los últimos años están siendo muy activos en lo que se refiere al desarrollo de iniciativas que promueven un avance en la igualdad de género efectiva en las empresas, donde aún se constatan brechas en diferentes áreas (Hutchinson et al., 2017; Grant Thornton, 2017), por lo que aún queda mucho camino por recorrer (Compromiso Empresarial, 2017). En las últimas décadas también ha aumentado la importancia de la información no financiera, ya sea para ampliar la información financiera tradicional, o para informar sobre los impactos que genera la actividad de las orgaizaciones para sus grupos de interés (Moser y Martin, 2012), tanto en términos de RSC (Moneva y Llena, 1996), como en el ámbito de los Objetivos para el Desarrollo Sostenible (ODS), a nivel mundial, y Europa, en particular (UE, 2013; CE, 2016).

En concreto, la Unión Europea (UE, 2014a), que señala la divulgación de información social y medioambiental como área prioritaria para fortalecer la atención de los negocios hacia la RSC, en 2014 publicó la Directiva 2014/95 (UE, 2014b) sobre divulgación de información no financiera e información sobre diversidad para ciertas grandes entidades y grupos de más de 500 personas empleadas, que la aplicarán en 2018 , porque se espera que una mayor transparencia logre que las empresas rindan mejor y sean más resilientes, tanto en términos financieros como no financieros. Siguiendo el contenido normativo de la nueva directiva, las entidades cotizadas deben incluir, además, en su Informe de Gobierno Corporativo, una descripción de la política de diversidad en relación con los órganos de administración, gestión y supervisión relativa a aspectos como por ejemplo, la edad, la formación académica, experiencia profesional, o el género; los objetivos de dicha política; cómo ha sido implementada, los resultados en el periodo del que se informa. Y si no se aplicara, el Informe incluirá una explicación de por qué es el caso.

En España, aunque con retraso, se ha aprobado de forma urgente el Real Decreto-ley 18/2017, que modifica el artículo 49 del Código de Comercio, así como los artículos 262 y 540 de la Ley de Sociedades de Capital. El RDL 18/2017 establece que las Entidades de Interés Público en España presentarán un Estado No Financiero (individual o consolidado) si el número medio de personas empleadas (o del grupo) es superior a 500, y si durante dos ejercicios consecutivos se cumplen dos de estas tres circunstancias: a) Activo total (o consolidado) superior a 20.000.000 euros; b) Importe Neto de la cifra de Negocios (o consolidada) supere los 40.000 .000 euros; c) Número medio de personas empleadas de la entidad (o de la dominante) sea superior a doscientos cincuenta.

También con retraso sobre los plazos inicialmente previstos, la Comisión Europea aprobó en julio de 2017 la Comunicación «Guidelines on non-finan- 
cial reporting (methodology for reporting non-financial information)» (CE, 2017b). Con una metodología basada en principios que sirva para empresas de todos los sectores de actividad, la guía se ha basado en las mejores prácticas y desarrollos relevantes que se están produciendo tanto a nivel de la UE como a nivel internacional. Entre los ejemplos de Indicadores Clave de Resultados que una entidad podría considerar divulgar, destacamos los que figuran en relación con la diversidad de género como son el derecho al permiso parental, por sexo; la temporalidad laboral, por sexo; y el promedio de horas de formación por persona empleada, por sexo.

En cuanto a la divulgación sobre la diversidad del Consejo de Administración, la orientación específica para ayudar a las grandes compañías cotizadas que deberán preparar una descripción de su política de diversidad del Consejo como parte de su Informe de Gobierno Corporativo a partir de 2018, propone que la política debería informar sobre los criterios que se aplican para garantizar que el Consejo tiene una diversidad suficiente de puntos de vista y experiencia necesaria para una buena comprensión de los asuntos actuales, así como los riesgos y oportunidades a lo largo plazo relacionados con el negocio; la razón para elegirlos; los objetivos específicos medibles advirtiendo que es particularmente útil establecer objetivos cuantitativos y marcos temporales, en particular, respecto del equilibrio de género; la implantación de políticas de diversidad en el plan de sucesión, la selección, la nominación y la evaluación; el rol de los comités competentes en estos procesos; si se ha proporcionado esta información a las personas y entidades accionistas en el momento de elegir o renovar las personas miembros del Consejo, cuando sea relevante; y el estado de la implementación y resultados desde el último informe para todos los aspectos de diversidad. Si los objetivos de diversidad no se han cumplido, la empresa debería divulgar cómo pretende cumplir los objetivos, incluyendo el marco de tiempo esperado en el que dichos objetivos deben cumplirse. Así, observamos que, al menos las empresas cotizadas, se enfrentan a un reto importante en materia de información sobre diversidad de género en sus órganos de gobierno.

Entre otras iniciativas recientes, la Guía Esencial para la Contabilidad Social y del Capital Humano (A4S, 2017) preparada con participación de personal directivo de Siemens, Burberry o Sainsbury's, entre otras, recuerda que el rol del equipo financiero es clave para asegurar que el modelo financiero puede ser validado y soportado en evidencias, incluso asegurado externamente, y presenta las áreas clave que, en su experiencia, se encuentran en el ámbito de las grandes definiciones sobre capital humano y social, explicando que las organizaciones pueden identificar tanto impactos como dependencias entre ambos. En este sentido, entendemos que en lo que respecta a la variable género (la incorporación de las mujeres), produce impactos, por una parte, en la «fuerza y resiliencia de la fuerza de trabajo", en la medida que algunas de las capacidades de que dispongan las mujeres sean diferentes de las de los hombres, y especialmente en el área 
"condiciones de trabajo» de las entidades, en el sentido de que aumenta su "diversidad», pero que deben cuidarse las áreas de «Reconocimiento y remuneración justa», "Derechos laborales", "Seguridad y salud», y en definitiva, "Bienestar» de todas las personas, para que no se produzcan discriminaciones o brechas entre unas y otros. En todo caso, en dicha guía (A4S, 2017) no hemos encontrado ningún ejemplo de información sobre políticas de capital humano relativos a la diversidad de género, esto es, de objetivos que se hayan planteado (con sus correspondientes criterios a aplicar, procedimientos de verificación, etc.) para aumentar la diversidad y sus relaciones con los procesos de selección, formación, remuneración, etc. de las mujeres empleadas.

Por lo tanto, observamos que la Unión Europea está concienciada de que las empresas deben integrar la gestión de la diversidad (incluyendo la de género) en su estrategia de negocio y de gobernanza, por los beneficios que se generan tanto socialmente como para la propia organización, y que las entidades deben informar adecuadamente sobre ello. Por eso creemos que es necesario continuar trabajando específicamente para lograr una divulgación de calidad sobre la diversidad de género en las empresas (relevante, comparable $y$, en definitiva, que resulte de utilidad para las diferentes partes interesadas), que hasta el presente no se ha abordado suficientemente ni en la literatura contable ni en la práctica de las empresas y otro tipo de organizaciones.

\section{Referencias bibliográficas}

AECA (2012). Propuesta de documento AECA: Información Integrada - Integrated Reporting. Cuadro Integrado de Indicadores (CII-FESG) y su Taxonomía XBRL. Ponentes del documento: Francisco Flores, José Luis Lizcano, María Mora y Manuel Rejón. Presentado en Madrid, el 26 de abril.

A4S CEO Leadership Network (2017). Essencial guide to Social and Human Capital Accounting. Accounting for Sustainability. https://www.accountingforsustainability.org/ social-and-human-capital (fecha de acceso 16 de noviembre de 2017).

Aranguren, Nagore y OchoA, Elena (2008). «Divulgación de información sobre empleados y medio ambiente en España y Alemania: una nota de investigación». Revista de contabilidad. Vol. 11 Núm. 2, 123-142. http:/www.rc-sar.es/verPdf. php?articleId $=182$

Archel, Pablo (2003). «La divulgación de la información social y medioambiental de la gran empresa española en el periodo 1994-1998: situación actual y perspectivas». $R e-$ vista Española de Financiación y Contabilidad. 38(117), 571-599. http://aeca.es/pub/ refc/articulos.php?id=0086

Carmona, Salvador y Carrasco, Francisco (1988). «Información de contenido social y estados contables. Una aproximación empírica y algunas consideraciones teóricas». Actualidad Financiera, noviembre, 2175-2192.

CE (Comisión Europea) (2016). Next steps for a sustainable European future. European action for sustainability. 739 Final Communication From The Commission To The Eu- 
DiVERSIDAd DE GÉNERO COMO PARTE DE LA RSC y SOSTENIBILIDAD EN LAS EMPRESAS. AVANCES PARA LA MEJORA

ropean Parliament, The Council, The European Economic And Social Committee And The Committee Of The Regions. 22.11.2016 COM(2016). Strasbourg, https:// ec.europa.eu/europeaid/sites/devco/files/communication-next-steps-sustainable-europe-20161122_en.pdf (fecha de acceso 17 de noviembre de 2017).

CE (Comisión Europea) (2017a). Guidelines on non-financial reporting (methodology for reporting non-financial information). Communication From The Commission Draft. 26.06.2017. Brussels. http://www.comunicarseweb.com.ar/sites/default/files/170627communication-non-financial-reporting-guidelines_en.pdf (fecha de acceso 17 de noviembre de 2017).

CE (Comisión Europea) (2017b). Comunicación de la Comisión. Directrices sobre la presentación de informes no financieros (Metodologia para la presentación de información no financiera). DOUE, 5 de julio, 2017/C, 215/01. http://eur-lex.europa.eu/legalcontent/ES/TXT/PDF/?uri=CELEX:52017XC0705(01)\&from=ES (fecha de acceso 28 de noviembre de 2017).

CNMV (Comisión Nacional del Mercado de Valores) (2013). Guia para la elaboración del informe de gestión de las entidades cotizadas. Julio. http://www.cnmv.es/DocPortal/ Publicaciones/Grupo/Guia_Gral.pdf

Compromiso_Empresarial (2017). «España, entre los 25 países que mejor cumplen los ODS, aunque baja en igualdad». 13 de Julio. https://www.compromisoempresarial. com/tercersector/2017/07/espana-entre-los-25-paises-que-mejor-cumplen-los-odsaunque-baja-en-igualdad/

Dobija, Mieczyslaw (1998). "How To Place Human Resources Into The Balance Sheet?». Journal of Human Resource Costing \& Accounting. 3(1), 83-92. https://doi. org/10.1108/eb029044

Gamerschlag, Ramin y Moeller, Klaus (2011). «The positive effects of human capital reporting. Corporate Reputation Review». 14(2), 145-155. https://link.springer.com/ article/10.1057/crr.2011.11

García-Meca, Emma (2008). «Información sobre capital humano revelada a analistas financieros». Partida Doble, Núm. 196, 76-88.

Ghasempour, Abdolreza y Yusof, Mohd Atef Md (2014). "Quality of Intellectual Capital and Human Resources Disclosure on the Firm Valuation». Open Journal of Accounting, Núm. 3, 59-70. http://www.scirp.org/journal/PaperInformation. aspx?paperID $=44740$

Grant, Thornton (2017). Women in business 2017. http://www.grantthornton.es/ perspectivas/ibr/women-in-business-2017/

HM Treasury (2017). Women in Finance Charter. A pledge for gender balance across financial services. 10 de noviembre. https:/www.gov.uk/government/uploads/system/ uploads/attachment_data/file/519620/women_in_finance_charter.pdf (fecha de acceso 21 de noviembre de 2017).

Hutchinson, Marion R.; Mack, Janet y Verhoeven, Peter (2017). «Women in Leadership: An Analysis of the Gender Pay Gap In ASX-Listed Firms». Accounting \& Finance, 57(3), 789-813, http://dx.doi.org/10.1111/acfi.12178 (fecha de acceso 28 de noviembre de 2017).

ICAC (2002). Informe sobre la situación actual de la contabilidad en España y líneas básicas para abordar su Reforma (Libro Blanco para la reforma de la contabilidad en España). ICAC, Madrid. http://www.icac.meh.es/Documentos/Contabilidad/1. Nacional/07.Otra\%20documentacion/2.Otros/10.Libro\%20Blanco\%20de $\% 20$ la\%20Contabilidad.pdf 
KNAuER, Thorsten (2010). «Relevance, quality and determinants of human capital disclosure -an empirical analysis of the German HDAX companies»e«The narrative employee reporting in Finland. Constructing the common goal of continuous growth and development». 33 Congreso EAA, Estambul, Turquía.

Marqués, E. (1974). La comptabilité des ressources humaines. Ed. Hommes et techniques.

Martínez, Aurora y Burgos, María Concepción (2010). "La contabilidad del Capital Humano: lecciones aprendidas y desafíos futuros». Revista de Contabilidad y Tributación. CEF. 334, 127-180.

Martínez Churiaque, José Ignacio (1978): La contabilidad de los recursos humanos: una nueva dimensión del sistema informativo empresarial. Tesis doctoral, Univesidad de Bilbao.

Martínez Churiaque, José Ignacio (1980): Estados contables con datos de inversión en personal. Su impacto sobre la toma de decisiones, Boletín de Estudios Económicos, n. ${ }^{\circ} 109$, pp. 127-147, Bilbao.

Meritum (1998). Measuring Intangibles to Understand and Improve Innovation Management. Proposal for a cost-shared RTD project. TSER European Programme. IVFramework Programme.

Meritum Project (2002). Guidelines for managing and reporting on intangibles. Fundación Arital Móvil. Madrid.

Moneva, José Mariano y Llena, Fernando (1996). «Análisis de la información sobre responsabilidad social en las empresas industriales que cotizan en bolsa». Revista Española de Financiación y Contabilidad, 25(87), 361-401

Moneva, José Mariano; Garayar, Ainhoa y Álvarez, Igor (2014). «Principales novedades en la nueva generación de memorias de sostenibilidad de la Global Reponrting Initiative (G4)». Revista aeca. 106 (junio), 35-38. Madrid: Asociación Española de Contabilidad y Administración de Empresas.

Moser, Donald V. y Martin, Patrick R. (2012). "A Broader Perspective on Corporate Social Responsibility Research in Accounting». The Accounting Review 87(3), 797 806. DOI: $10.2308 /$ accr-10257

Pons, Ángela (1996). «La información social sobre empleados». Partida Doble. Núm. 73, 23-29.

SAINZ, J. (1990). "La auditoría social». Nuevas tendencias de gestión de recursos humanos, 179-196. Universidad de Deusto, Bilbao.

SaItua, Ainhoa (2010). "Análisis de la divulgación sobre recursos, riesgos y relaciones clave en el informe de gestión: una propuesta metodológica». Revista Iberoamericana de Gestión 16(4), 1-18. http://www.observatorio-iberoamericano.org/RICG/ N\%C2\%BA_16/Ainhoa_Saitua_Iribar.pdf

Saitua, Ainhoa; Andicoechea, Lorea; Albizu, Eneka (2014a). "Human resources information in the Management Report of the top Spanish companies». $2^{\text {nd }}$ World Conference On Business, Economics And Management - WCBEM 2013 http://www.sciencedirect. com/science/article/pii/S1877042813050714

Saitua, Ainhoa; Andicoechea, Lorea y Albizu, Eneka (2014b). «Divulgación sobre recursos humanos en el Informe de Gestión. Análisis del nivel de comparabilidad y uso de indicadores en las empresas del IBEX 35». Boletín de Estudios Económicos 69 (213), $549 \mathrm{https://search.proquest.com/docview/1655115952?pq-origsite=gscholar} \mathrm{(fecha} \mathrm{de}$ acceso 17 de noviembre de 2017).

Saitua, Ainhoa; Andicoechea, Lorea y Albizu, Eneka (2014c). «El informe integrado: una propuesta de encaje para la información sobre capital humano", Perspectiva Em- 
DiVERSIDAd DE GÉNERO COMO PARTE DE LA RSC y SOSTENIBILIDAD EN LAS EMPRESAS. AVANCES PARA LA MEJORA

presarial, 1(1). 33-47. http://rpe.ceipa.edu.co/index.php/perspectiva-empresarial/article/view/10 (fecha de acceso 17 de noviembre de 2017).

Saitua, Ainhoa; Moya, Soledad y Vázquez, Isabel (2015). "Informe de gestión de empresas cotizadas. Valoración crítica de la guía de la CNMV y propuesta para analizar la información divulgada». Revista de dirección y administración de empresas = Enpresen zuzendaritza eta administraziorako aldizkaria, ISSN-e 2341-0108, 22(46-64) https:// addi.ehu.es/bitstream/handle/10810/16765/46.pdf?sequence $=1$ \&isAllowed=y (fecha de acceso 17 de noviembre de 2017).

Sierra Molina, Guillermo Juan y Moreno Campos, Inés (2000). «La información contable sobre el capital humano». Partida doble, ISSN 1133-7869, Núm. 116, 60-71.

UE (Unión Europea) (2013). Report on corporate social responsibility: accountable, transparent and responsible business behaviour and sustainable growth $(2012 / 2098$ INI), 28 enero. http://www.europarl.europa.eu/sides/getDoc.do?pubRef=-//EP// TEXT+REPORT+A7-2013-0017+0+DOC+XML+V0//EN (fecha de acceso 17 de noviembre de 2017).

UE (Unión Europea) (2014). Corporate Social Responsibility. National Public policies in the European Union. Compendium 2014.

Villacorta, Miguel Ángel (2006). Revelación de la información voluntaria sobre el capital humano en los informes anuales. Intangible Capital, Núm. 2, 37-71. https://upcommons.upc.edu/bitstream/handle/2099/2932/Revelaci\%C3\%B3n\%20 de\%20la\%20informaci\%C3\%B3n\%20voluntaria\%20sobre\%20el\%20capital\%20 humano\%20en\%20los\%20informes\%20anuales.pdf (fecha de acceso 17 de noviembre de 2017).

Wyatt, A. y Frick, H. (2010). Accounting for investments in human capital: A review. Australian Accounting Review. Vol. 20 Núm. 3, 265-273.

\section{Normativa europea}

Directiva 2003/51/CE del Parlamento Europeo y del Consejo, de 18 de junio de 2003, sobre las cuentas anuales y consolidadas de determinadas formas de sociedades, bancos y otras entidades financieras y empresas de seguros (Directiva de Modernización).

Directiva 2013/34/UE del Parlamento Europeo y del Consejo de 26 de junio de 2013 sobre los estados financieros anuales, los estados financieros consolidados y otros informes afines de ciertos tipos de empresas, por la que se modifica la directiva 2006/43/CE del Parlamento Europeo y del Consejo y se derogan las Directivas 78/660/CEE y 83/349/CEE del Consejo, https://www. boe.es/doue/2013/182/L00019-00076.pdf

Directiva 2014/95/UE del Parlamento Europeo y del Consejo, de 22 de octubre de 2014, por la que se modifica la Directiva 2013/34/UE en lo que respecta a la divulgación de información no financiera e información sobre diversidad por parte de determinadas grandes empresas y determinados grupos. DOUE 330, de 15 de noviembre de 2014. https://www.boe.es/diario_boe/txt. php?id=DOUE-L-2014-83373 (fecha de acceso 17 de noviembre de 2017). 


\section{Normativa española}

Ley 19/1989, de 25 de julio, de reforma parcial y adaptación de la legislación mercantil a las Directivas de la comunidad Económica Europea (CEE) en materia de Sociedades. https://publications.europa.eu/es/publication-detail//publication/4b4a658d-c0bb-4658-8459-406da3279f61/language-es

Ley 62/2003, de 30 de diciembre, de medidas fiscales, administrativas y del orden social. https://www.boe.es/buscar/doc.php?id=BOE-A-2003-23936

Real Decreto Legislativo 1/2010, de 2 de julio, por el que se aprueba el texto refundido de la Ley de Sociedades de Capital. BOE núm. 161, de 03/07/2010 https://www.boe.es/buscar/act.php?id=BOE-A-2010-10544

Real Decreto-ley 18/2017, de 24 de noviembre, por el que se modifican el Código de Comercio, el texto refundido de la Ley de Sociedades de Capital aprobado por el Real Decreto Legislativo 1/2010, de 2 de julio, y la Ley 22/2015, de 20 de julio, de Auditoría de Cuentas, en materia de información no financiera y diversidad. BOE de 25 de noviembre de 2017. http:/www.boe.es/diario_boe/txt. php?id=BOE-A-2017-13643

\section{ANEXO. Listado de marcos internacionales que la Comisión Europea ha tomado como referencia para la elaboración de la Guía sobre información no financiera (CE, 2017b, p. 3)}

- el CDP (antiguo Carbon Disclosure Project, proyecto de divulgación de las emisiones de carbono),

- la Junta de Normas de Divulgación del Clima,

- las Directrices de la OCDE sobre la diligencia debida para la gestión responsable de las cadenas de suministro procedentes de zonas afectadas por conflictos y zonas de alto riesgo, y sus suplementos,

— el Sistema de Gestión y Auditoría Medioambientales (EMAS) y los documentos de referencia sectoriales conexos,

- Los indicadores clave de resultados ambientales, sociales y de gobernanza de la Federación Europea de Asociaciones de Analistas Financieros: una directriz para la integración de los factores ambientales, sociales y de gobernanza en el análisis financiero y la valoración empresarial,

- la Iniciativa Mundial de Presentación de Informes, Global Reporting Initiative (GRI)

— las Directrices de la OCDE-FAO para las cadenas de suministro agrícolas responsables,

- las Directrices sobre elaboración de informes del Consejo de presentación de informes financieros del Reino Unido (Guidance on the Strategic Report of the UK Financial Reporting Council), 
- las Líneas Directrices de la OCDE para Empresas Multinacionales,

- el marco de presentación de informes de los Principios Rectores sobre las empresas y los derechos humanos,

— la norma ISO 26000 de la Organización Internacional de Normalización,

- el Marco internacional información integrada (IR),

- las Directrices modelo para la presentación de información sobre los factores ambientales, sociales y de gobernanza a los inversores, de la Iniciativa Bolsas de Valores Sostenibles de las Naciones Unidas,

- el Protocolo del Capital Natural,

- las Guías sobre la huella ambiental de los productos y las organizaciones,

- la Junta de normas de contabilidad sostenible (Sustainability Accounting Standards Board),

— el Código de sostenibilidad del Consejo Alemán para el Desarrollo Sostenible,

- la Declaración tripartita de principios sobre las empresas multinacionales y la política social, de la Organización Internacional del Trabajo (OIT),

- el Pacto Mundial de las Naciones Unidas,

- los Objetivos de Desarrollo Sostenible (ODS) de las Naciones Unidas, resolución de 25 de septiembre de 2015, «Transformar nuestro mundo: la Agenda 2030 para el Desarrollo Sostenible»,

- los Principios Rectores de las Naciones Unidas sobre las empresas y los derechos humanos: puesta en práctica del marco de las Naciones Unidas para «proteger, respetar y remediar». 